\title{
Optimized Speech Compression Algorithm Based on Wavelets Techniques and its Real Time Implementation on DSP
}

\author{
Noureddine Aloui \\ Sciences Faculty of Tunis, Innov'COM Laboratory, University of Tunis El-Manar, 2092, Tunisia \\ Email: aloui.noureddine@gmail.com \\ Souha Bousselmi \\ Sciences Faculty of Tunis, Innov'COM Laboratory, University of Tunis El-Manar, 2092, Tunisia \\ Email: bousselmi.souha2008@gmail.com \\ Adnane Cherif \\ Sciences Faculty of Tunis, Innov'COM Laboratory, University of Tunis El-Manar, 2092, Tunisia \\ Email: adnane.cher@fst.rnu.tn
}

\begin{abstract}
This paper presents an optimized speech compression algorithm using discrete wavelet transform, and its real time implementation on fixed-point digital signal processor (DSP). The optimized speech compression algorithm presents the advantages to ensure low complexity, low bit rate and achieve high speech coding efficiency, and this by adding a voice activity detector (VAD) module before the application of the discrete wavelet transform. The VAD module avoids the computation of the discrete wavelet coefficients during the inactive voice signal. In addition, a real-time implementation of the optimized speech compression algorithm is performed using fixed-point processor. The optimized and the original algorithms are evaluated and compared in terms of CPU time (sec), Cycle count (MCPS), Memory consumption (Ko), Compression Ratio (CR), Signal to Noise Ratio (SNR), Peak Signal to Noise Ratio (PSNR) and Normalized Root Mean Square Error (NRMSE).
\end{abstract}

Index Terms - Speech Compression, Discrete Wavelet Transform, Voice Activity Detection, Hardware Implementation, Digital Signal Processor, RTW

\section{INTRODUCTION}

During last decade, the wavelet transform has emerged as a powerful and robust mathematical tool for analyzing non-stationary signal, and has been used in wide range of signal processing applications such as: speech, image and video compression [1][2][3], speech denoising [4], pattern recognition [5] and electrocardiogram (ECG) signal processing [6]. Recently many new algorithms have been developed based on wavelet transform [7] [8] [9] or wavelet packets transform [10] [11] to compress speech signals.

The speech compression techniques using discrete wavelet transform (DWT) achieves a high compression ratio and acceptable reconstructed signal, compared to other existing techniques such as; DCT (Discrete Cosine Transform) used by the MPEG standard [7], FFT (Fast Fourier Transform) [7] [12], LPC (Linear Predictive
Coding) for the NATO (North Atlantic Treaty Organization) and GSM (Global System Mobile) [13] or the CELP (Code Excited Linear Prediction) [14] for the International telecommunication Union (UIT). However, the main disadvantage of the speech compression using DWT is linked to the high complexity of implementation, during the real time processing on a DSP processor. In this context, this paper presents an optimized algorithm for speech compression based on DWT and its real-time implementation on fixed point DSP for Texas Instruments.

The paper is organized as follows: Section 2 discusses the overview of analysis and synthesis using wavelets. Section 3 presents the methodology of the speech compression algorithm based on DWT and discusses the optimized speech compression algorithm. In addition comparative study of performances is performed. In section 4, a real-time implementation methodology of the optimized speech compression algorithm is presented and a comparative study of performances is performed using some objective criteria. Section 5, gives a conclusion and some remarks.

\section{ANALYSIS AND SYNTHESIS USING WAVELETS}

\section{A. Continuous Wavelet Transform (CWT):}

The concept of CWT was introduced by A. Grossman and J. Morlet [15] [16], to analyze the seismic signals structures at different scales. The principal of CWT consists in decomposing the original signal in too many functions by using a single prototype function called mother wavelet $(\psi(t))$.

The CWT of a signal $f(t)$ is computed using the following formula: 


$$
W_{f}(a, b)=\frac{1}{\sqrt{|a|}} \int_{-\infty}^{+\infty} f(t) \psi^{*}\left(\frac{t-b}{a}\right) d t
$$

where $(*)$ denotes the complex conjugate, $a$ and $b$ present respectively the dilation factor and the translation factor of the mother wavelet:

$$
\psi_{(a, b)}(t)=|a|^{-\frac{1}{2}} \psi\left(\frac{t-b}{a}\right) \quad a, b \in \mathbb{R} ; a \neq 0
$$

The inverse continuous wavelet transform (ICWT) is obtained by applying the following formula:

$$
f(t)=\frac{1}{C_{\psi}} \int_{a=-\infty}^{+\infty} \int_{b=-\infty}^{+\infty} \frac{1}{|a|^{2}} W_{f}(a, b) \frac{1}{\sqrt{|a|}} \psi\left(\frac{t-b}{a}\right) d a d b
$$

where $C_{\psi}$ is a constant that depends on the mother wavelet.

\section{B. Discrete Wavelet Transform $(D W T)$ :}

The DWT is a special case of CWT; it was developed to apply the CWT to the digital world. It obtained by discretizing $a$ and $b$ factors as follows:

$$
W_{\psi} f(m, n)=\int_{-\infty}^{+\infty} f(t) \psi_{(m, n)}^{*}(t) d t
$$

where $\psi_{(m, n)}(t)=2^{-m} \psi\left(2^{m} t-n\right), \quad m, n \in \mathbb{N}$

Generally, the DWT is computed using the recursive pyramid algorithm developed by $S$. Mallat [17]. It consists in successively decomposed the original signal into high frequency components and low frequency components respectively by the mean of the high-pass analysis filter $h_{1}(z)$ and low-pass analysis filter $h_{0}(z)$. The high frequency components (details coefficients) are not analyzed any further, however the low frequency components (approximation coefficients) are subsequently divided into new approximation coefficients and detail coefficients using the following formulas [17].

$$
\begin{aligned}
& Y_{\text {high }}[k]=\sum_{n=0}^{N-1} x(n) h_{1}(2 k-n) \\
& Y_{\text {low }}[k]=\sum_{n=0}^{N-1} x(n) h_{0}(2 k-n)
\end{aligned}
$$

where $Y_{h i g h}[k]$ and $Y_{l o w}[k]$ are the outputs of the highpass and low-pass filters obtained by down-sampling by a factor of 2 .

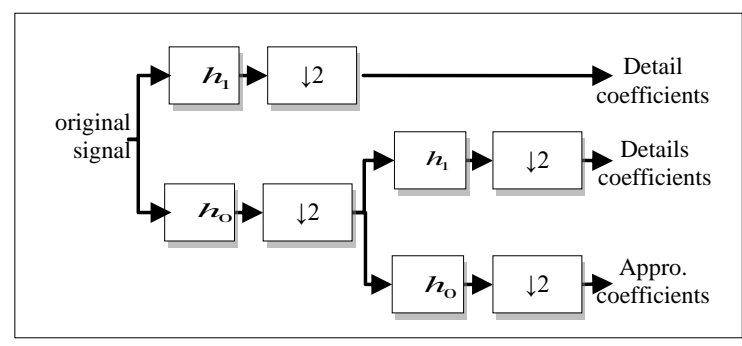

Fig. 1. Two level DWT analysis.

The original speech signal can be reconstructed using the inverse discrete wavelet transform (IDWT). In the first, the approximation and detail coefficients are upsampled by a factor of 2 , and then respectively filtered by the high-pass synthesis filter $g_{1}(z)$ and low-pass analysis filter $g_{0}(z)[17]$ :

$$
\begin{aligned}
& g_{1}(n)=h_{1}(N-n-1) \\
& g_{0}(n)=h_{0}(N-n-1)
\end{aligned}
$$

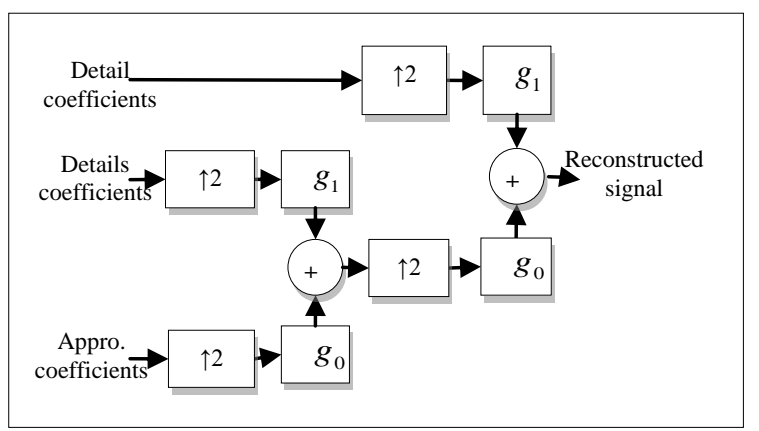

Fig. 2. Two level DWT synthesis.

\section{OPTIMIZED SPEECH COMPRESSION ALGORITHM USING DWT}

This section describes the optimized speech compression algorithm based on DWT. The first subsection details the principal of speech compression using DWT. The second subsection presents the optimized speech compression algorithm, by introducing a VAD module, and a comparative study of performances.

\section{A. Speech compression using wavelets}

The idea of speech compression using DWT is primarily related to the representation of a speech signal in the wavelet domain. In [18], W. Kinsner was shown that most of the wavelet coefficients have small amplitudes. More than $90 \%$ of the wavelet coefficients are less than $5 \%$ of the maximum value; this means that most of the signal energy is concentrated in the high valued coefficients, which are few [18]. Consequently, the speech compression is achieved by truncating the small valued coefficients and efficiently encoding them.

The speech compression based on DWT, can be performed in five steps, as shown in Fig. 3: in the first step, the original signal is decomposed into sub-bands by applying DWT. Then, the obtained sub-bands are thresholded and encoded. Finally, a quantization steps followed by entropy coding is applied: [7] [8] [9] [10] [12] [13] [18] [19]. The detailed steps of the speech compression using wavelets are:

- Step 1: This step consists in choosing the decomposition level, the mother wavelet and applying the DWT on the original speech signal, in order to compute wavelet coefficients. For choosing an optimal mother wavelet several criteria can be considered: the objective is minimizing the error variance and maximizing the signal to noise ratio between the original and reconstructed signal. Generally, the choice of the optimal mother wavelet is based on the average 


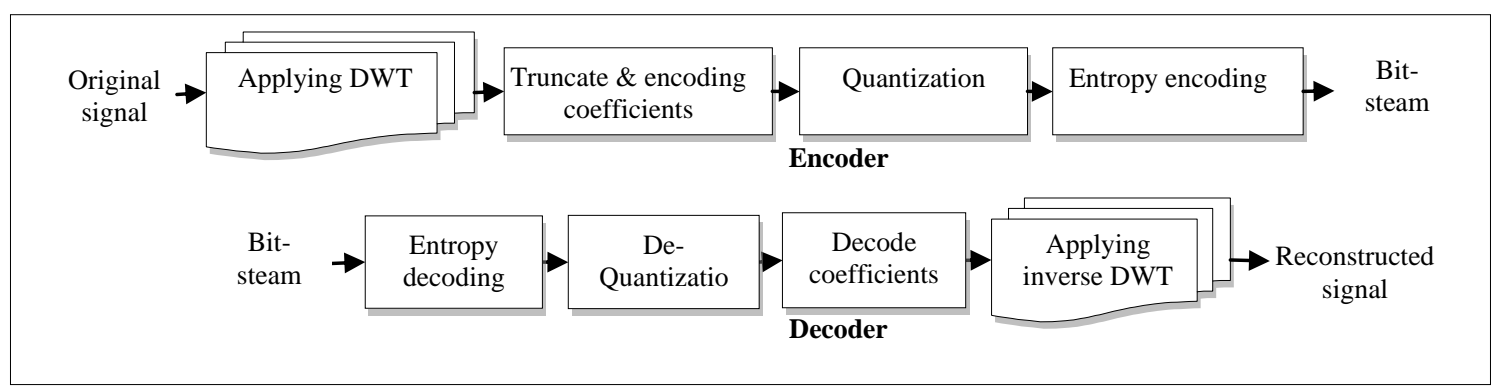

Fig. 3. Speech compression algorithm using DWT.

In [20] it was shown that the adequate decomposition level for speech compression should be less or equals to five, without further advantage gained with processing beyond scale five [20].

- Step 2: In this step, the obtained wavelet coefficients (sub-bands) are thresholded. Several methods exist for thresholding such as; the hard thresholding and the soft thresholding [21].

The hard and the soft thresholding for a given threshod value $(T>0)$ are performed using the following formulas:

- Hard thresholding:

$$
W_{\text {hard }}= \begin{cases}W & \text { if } \quad|W| \geq T \\ 0 & \text { Otherwise }\end{cases}
$$

- Soft thresholding:

$$
W_{\text {soft }}=\left\{\begin{array}{lll}
0 & \text { if } & |W| \leq T \\
\operatorname{sign}(W)(|W|-T) & \text { if } & W>T
\end{array}\right.
$$

where, sign and $W$ present respectively the sign function and the wavelet coefficients.

For calculating threshold values the thresholds values, there are many methods such as; the global threshold computation and the level dependent threshold computation:

- Global threshold:

$$
T=\left\{\begin{array}{l}
0.05 * \max \left(a b s\left(C_{d \mu_{\text {屏il }}}\right)\right) \text { if } \quad \sigma=0 \\
\sigma \quad \text { Otherwise }
\end{array}\right.
$$

where, $\sigma=\operatorname{median}\left\{a b s\left(C_{d \text { II }_{\text {Iil }} 1}\right)\right\}$ is the median of the absolute value of detail coefficients at level one.

- Level dependent threshold: can be calculated by the Birge-Massart strategy, using the following formula [22]:

$$
n_{i}=\frac{M}{(J+2-I)^{\alpha}}
$$

where $\alpha=1.5$ presents the compression parameter, $L \leq M \leq 2 * L$ and $\mathrm{L}$ is the length of approximation coefficients at level one.

- Step 3: The speech compression using DWT, is achieved by truncating the wavelet coefficients of low magnitude and efficiently encoding them. There are many ways to encode the truncated coefficients. One way is to represent the wavelet coefficients of high magnitude in their respective positions in the wavelet coefficients vector [12]. Another way is to encode the values of two consecutive zeros with two bytes; the first byte to indicate the start sequence of zeros in the wavelet coefficient vector and the second byte to representing the number of consecutive zeros [18]:

Let, $\quad x_{i}=\{3,2,0,0,0,0,5,1,0,0,0,0,0,1\} \quad$ a wavelet coefficient vector. The encoded vector $\left(x_{c}\right)$ is $x_{c}=\{3,2,0,4,5,1,0,5,1\}$.

- Step 4: Usually the quantization step precedes the entropy encoding, it has two objectives: extracting the pertinent information and facilitating the entropy encoding. The encoded coefficients for each sub-band are converted to others coefficients, with few possible discrete values. There are many quantization methods such as: uniform, scalar and vector quantization.

- Step 5: The quantized coefficients for each sub-band contains some redundancy, which waste of space. To remove it, an entropy coder like Huffman coding [10] or arithmetic coding is used.

At the decoder, the received bit-stream is used to decode (entropy decoding) and de-quantize (inverse quantization) the compressed sub-bands. Then, the decompressed sub-bands are decoded (Zero-value decoder) to obtain the sub-bands. Finally, the inverse discrete wavelet transform (IDWT) is applied to reconstruct speech signal.

\section{B. Optimized speech compression algorithm using wavelets}

In this subsection, a VAD module is added to the speech compression algorithm described in the previous subsection. However, the concept of VAD is widely used in the field of speech communication, in order to achieve high speech coding efficiency and low bit rate transmission. The VAD detector provides in its output a decision called a voice activity decision ( 0 or 1$)$; which is used to switch between the silence compression or DWT speech compression, as shown in Fig. 4. In addition, during inactive periods, the VAD module avoids the application of DWT to the speech signal.

Fig. 5 presents the speech compression algorithm described in the previous subsection with VAD detector. For each speech frame, the VAD detector module provides decision, which is used as to switch between the 
speech coding using DWT or silence coding. In addition, a bit allocation module can be used to optimize the number of bits used for quantizing the encoded wavelet coefficients. The bit allocation module is controlled by a psycho-acoustic model.

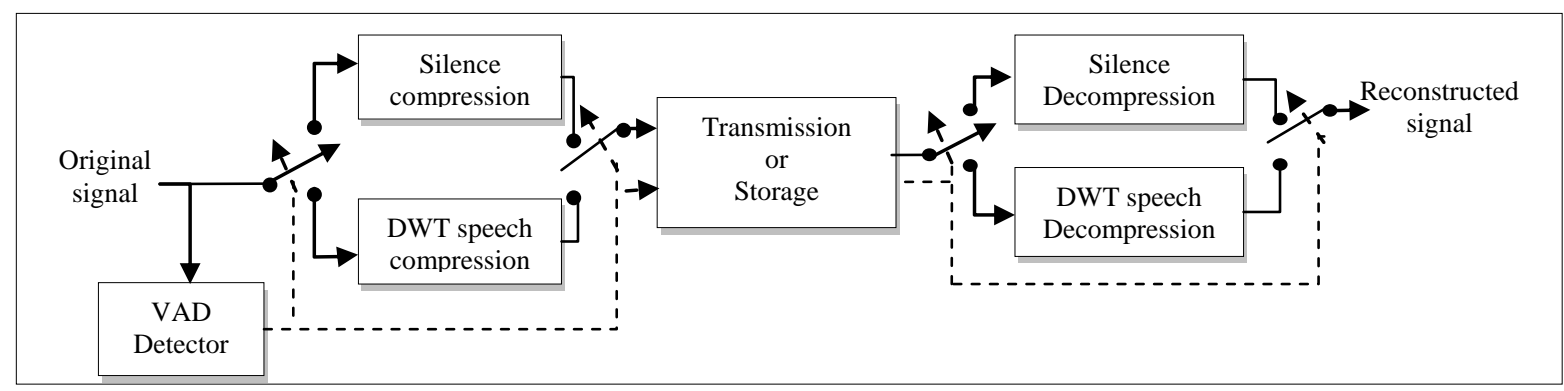

Fig. 4. Speech compression system with VAD.

There are many algorithms of VAD, such as; the algorithm described in [23], by Y. Ephram and D. Malah used in the MMSE-STSA (Minimum Mean-Square Error-
Short Time Spectral Amplitude) estimator for speech enhancement. In [24], A. Benyassine, described her VAD used in G.729B algorithm of the ITU [25].

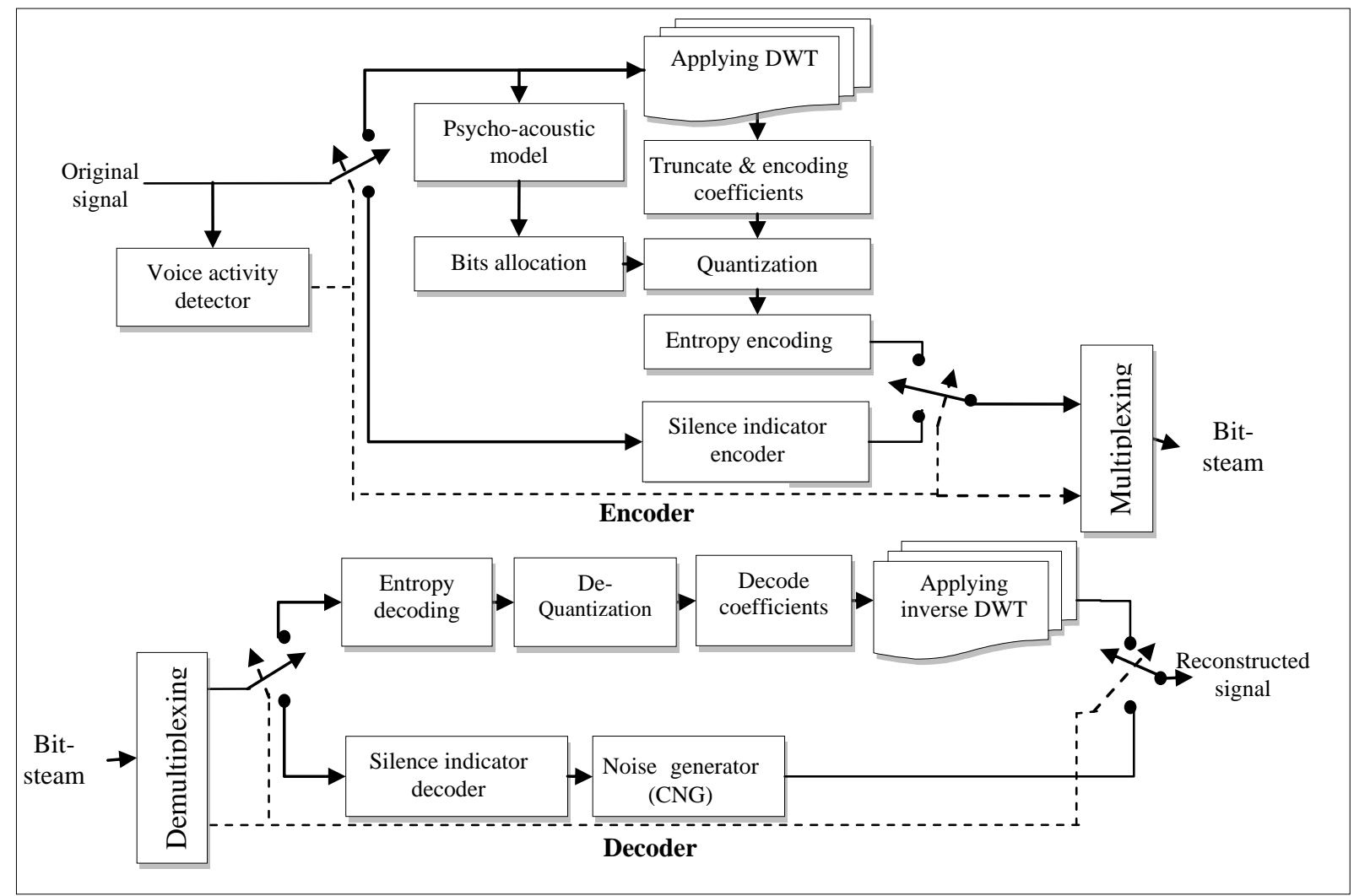

Fig. 5. Optimized speech compression algorithm using DWT.

Fig. 6 shows the speech signal "si2022.wav", extracted from the TIMIT database of Texas Instruments [26] and its VAD according to G.729B recommendation of the ITU.

Table 1 illustrates the compression performances with and without VAD module. The speech signals are extracted from the TIMIT database and analyzed by frame of 256 samples. The used mother wavelet is Daubechies "db10" and five decomposition levels.

From the above table, it is clear that the DWT speech compression algorithm with VAD module outperforms the DWT speech algorithm without VAD module in terms of CPU time, CR, SNR,

PSNR and NRMSE. We can also remark that the using of VAD module presents a very low-complexity and ameliorated performances if the speech signal contain a silence (Fig. 7). 


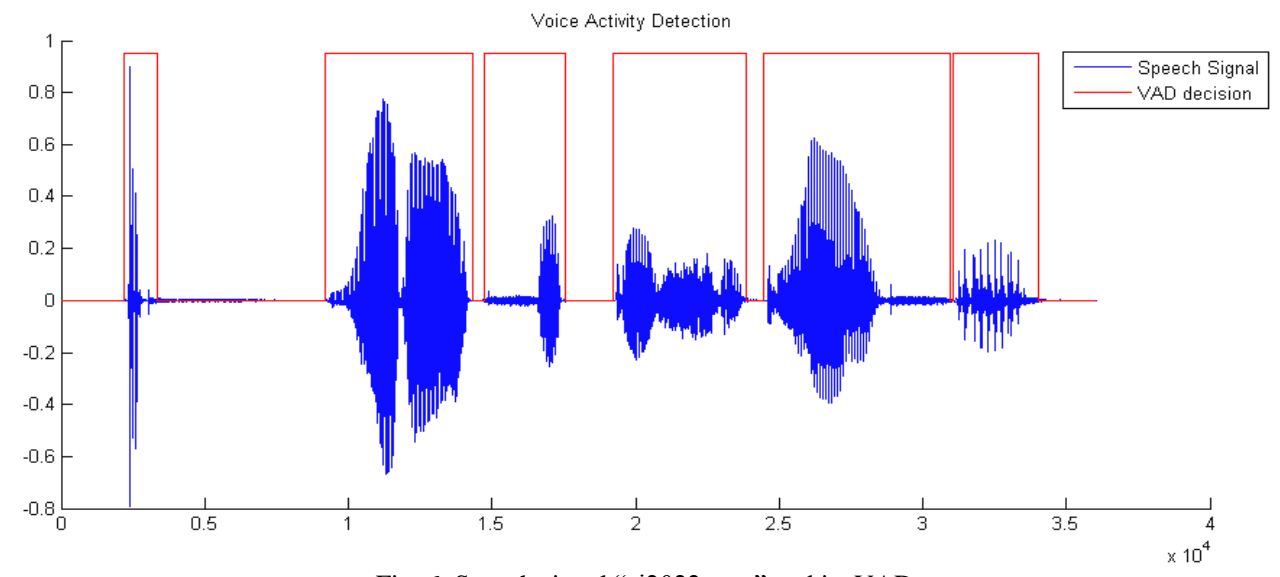

Fig. 6. Speech signal "si2022.wav" and its VAD

Table 1. Performance evaluation of the optimized algorithm using TIMIT speech files

\begin{tabular}{|c|c|c|c|c|c|c|}
\hline Speech TIMIT files & DWT speech algorithm & Average $\mathrm{CPU}$ time per frame & $\mathrm{CR}$ & SNR & PSNR & NRMSE \\
\hline \multirow{2}{*}{ sx20.wav } & Without VAD & 0.6234 & 1.2467 & 19.0936 & 36.4608 & 0.1110 \\
\hline & With VAD & 0.2128 & 1.8726 & 19.1289 & 36.4761 & 0.1109 \\
\hline \multirow{2}{*}{ sx21.wav } & Without VAD & 0.2141 & 1.8037 & 20.1918 & 38.7277 & 0.0978 \\
\hline & With VAD & 0.1360 & 2.1131 & 20.1286 & 38.6645 & 0.0985 \\
\hline \multirow{2}{*}{ sx22.wav } & Without VAD & 0.2058 & 1.7060 & 20.1277 & 37.8466 & 0.0985 \\
\hline & With VAD & 0.1056 & 2.0516 & 20.1921 & 37.9110 & 0.0978 \\
\hline \multirow{2}{*}{ sx23.wav } & Without VAD & 0.4787 & 1.1132 & 20.1214 & 41.9858 & 0.0986 \\
\hline & With VAD & 0.2943 & 1.3745 & 20.3202 & 42.1847 & 0.0964 \\
\hline \multirow{2}{*}{ sx24.wav } & Without VAD & 0.2500 & 1.2956 & 20.1325 & 37.5207 & 0.0985 \\
\hline & With VAD & 0.1266 & 1.8053 & 20.3011 & 37.6894 & 0.0966 \\
\hline \multirow{2}{*}{ sx25.wav } & Without VAD & 0.2001 & 1.7405 & 19.0468 & 37.6362 & 0.1116 \\
\hline & With VAD & 0.1221 & 2.0071 & 19.3803 & 37.9697 & 0.1074 \\
\hline \multirow{2}{*}{ sx26.wav } & Without VAD & 0.2031 & 1.6245 & 17.7602 & 35.0726 & 0.1294 \\
\hline & With VAD & 0.1294 & 2.0001 & 17.7359 & 35.0483 & 0.1298 \\
\hline \multirow{2}{*}{ sx27.wav } & Without VAD & 0.2034 & 1.8612 & 19.2385 & 40.1789 & 0.1092 \\
\hline & With VAD & 0.1171 & 2.1865 & 19.4345 & 40.3750 & 0.1067 \\
\hline \multirow{2}{*}{ sx28.wav } & Without VAD & 0.2717 & 1.6295 & 17.5460 & 37.2651 & 0.1326 \\
\hline & With VAD & 0.1953 & 1.8594 & 17.8291 & 37.5481 & 0.1284 \\
\hline \multirow{2}{*}{ sx29.wav } & Without VAD & 1.0791 & 1.7421 & 20.0023 & 47.0616 & 0.1000 \\
\hline & With VAD & 0.1537 & 3.8110 & 20.2572 & 47.3165 & 0.0971 \\
\hline \multirow{2}{*}{ sx30.wav } & Without VAD & 0.1749 & 1.5027 & 18.4965 & 39.1256 & 0.1189 \\
\hline & With VAD & 0.1193 & 1.7233 & 18.7768 & 39.4059 & 0.1151 \\
\hline
\end{tabular}

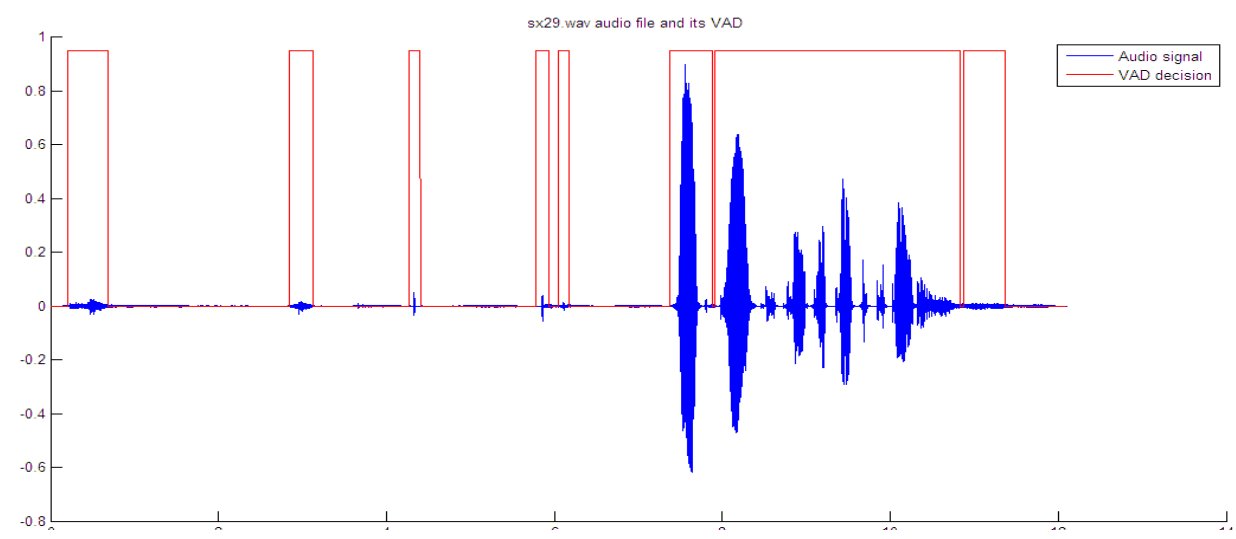

Fig. 7. Speech signal "sx29.wav" and its VAD 


\section{REAL-TIME IMPLEMENTATION METHODOLOGY}

In this section a real-time implementation of the optimized speech compression algorithm based on DWT is performed using the fixed point processor TMS320C6416 DSP. In addition, a comparative study of performances between the optimized version and the original version of DWT speech algorithms is presented using some objective criteria such as; the cycles count required to perform each algorithm and the memory sizes of DSP executable code.

For rapid development and real-time implementation of the speech compression algorithm based on DWT, the MATLAB/Simulink and the Real Time Workshop (RTW) tools are exploited. In the first step, the MATLAB/Simulink tool is used to design the speech codec, then the RTW is exploited to automatically generate a $\mathrm{C}$ code (ANSI-C) from the designed Simulink models. Finally the build process targeted the executable code to the DSP by the mean of Code Composer Studio (CCS) [27].

In this work, the original and the optimized speech compression algorithms are implemented using the DSP/BIOS tool. It is a scalable real time kernel, designed to be used by applications that require real-time scheduling and synchronization. It is also designed to minimize CPU requirements and memory on the target [28].

Fig. 8 presents the designed Simulink model for realtime implementation of the speech compression algorithm based on DWT using TMS320C6416 processor for Texas Instruments.

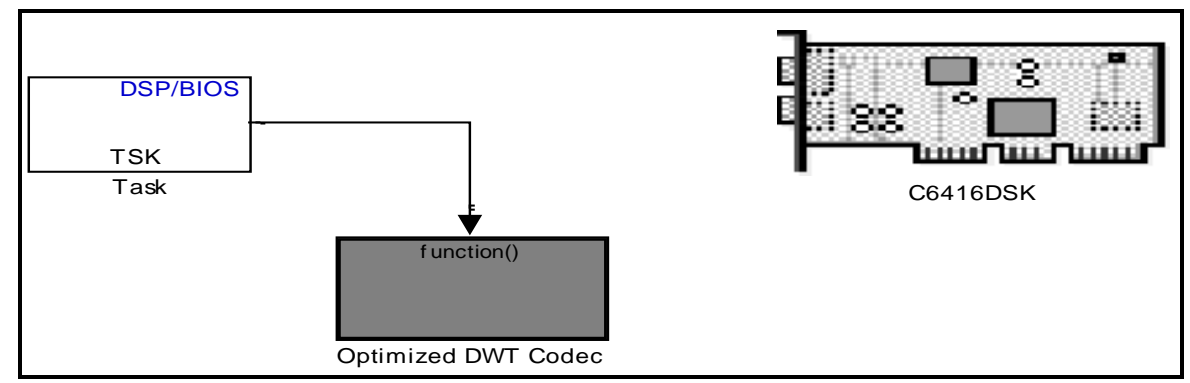

Fig. 8. Speech compression codec based on DWT using MATLAB/Simulink

Fig. 9 shows the Simulink model of the speech compression algorithm using DWT, which is implemented using a task TSK of DSP/BIOS. As it can be seen that the Simulink block "Optimized DWT Codec" is a subsystem block contains the different steps of the speech algorithm as shown in Fig.9.

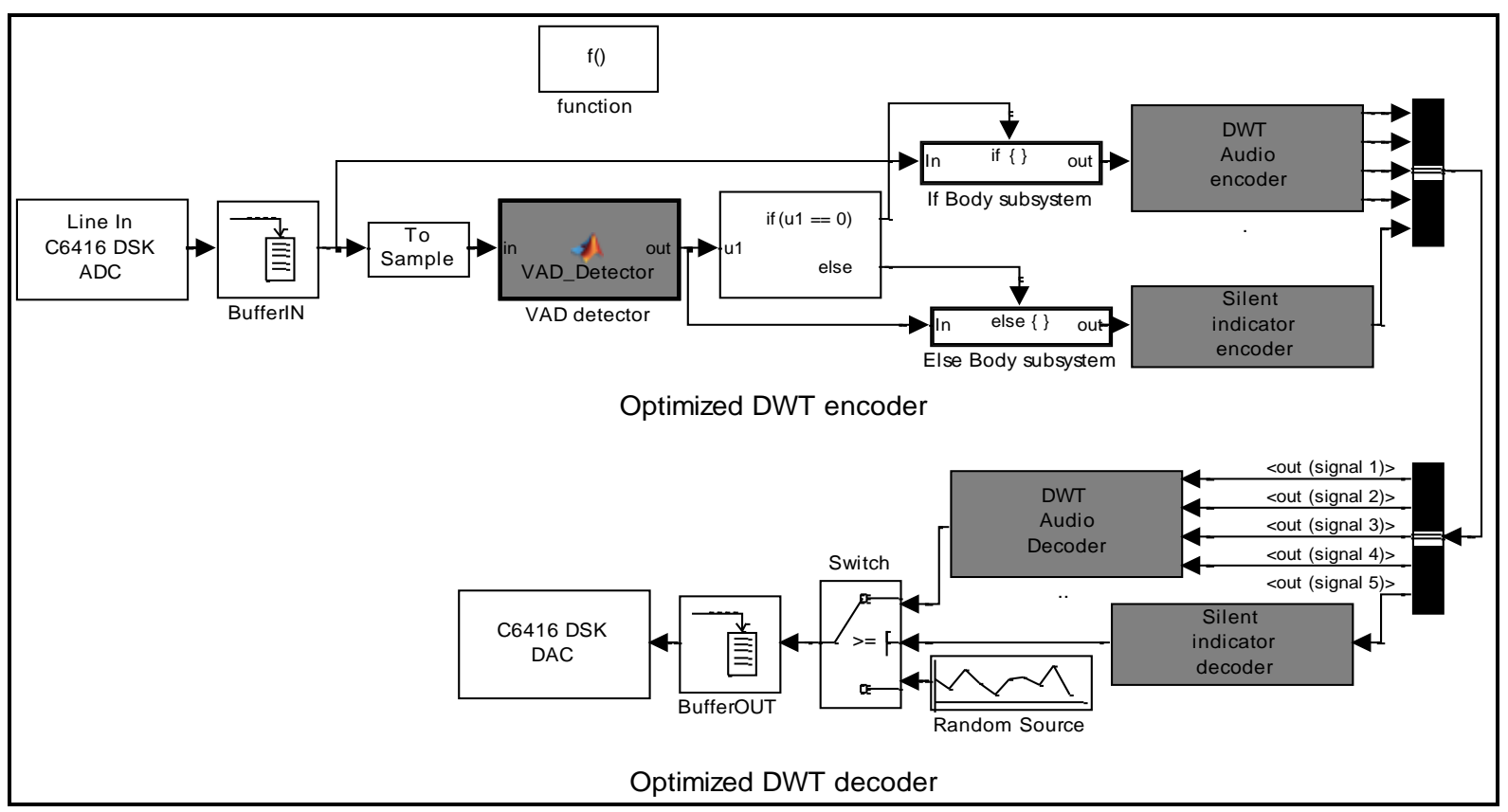

Fig. 9. Optimized speech compression algorithm based on DWT using MATLAB/Simulink

The DWT speech algorithm processes the analog speech signal from the microphone input or the line input of the codec AIC23, and then converted into a digital signal using an analog to digital converter (ADC). After the process of coding and decoding, the digital output signal from the DSP is converted to an analog signal using a digital to analog converter (DAC). Finally the speech data sent to the headphone output and the line output. The TMS320C6416 DSP communicates with the AIC23 codec via a bidirectional serial port McBSP2 


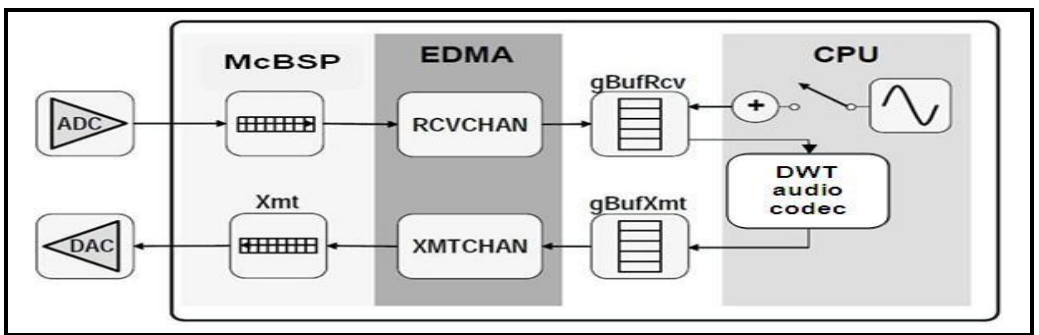

Fig. 10. Data flow in the hardware components of DSK 6416

As described in the previous section the DWT speech coding algorithm can be summarized in five steps:

1) Applying the DWT on the original speech signal.

2) Thresholding the obtained sub-bands.
3) Encoding the thresholded coefficients.

4) Quantization of encoded coefficients

5) Entropy encoding

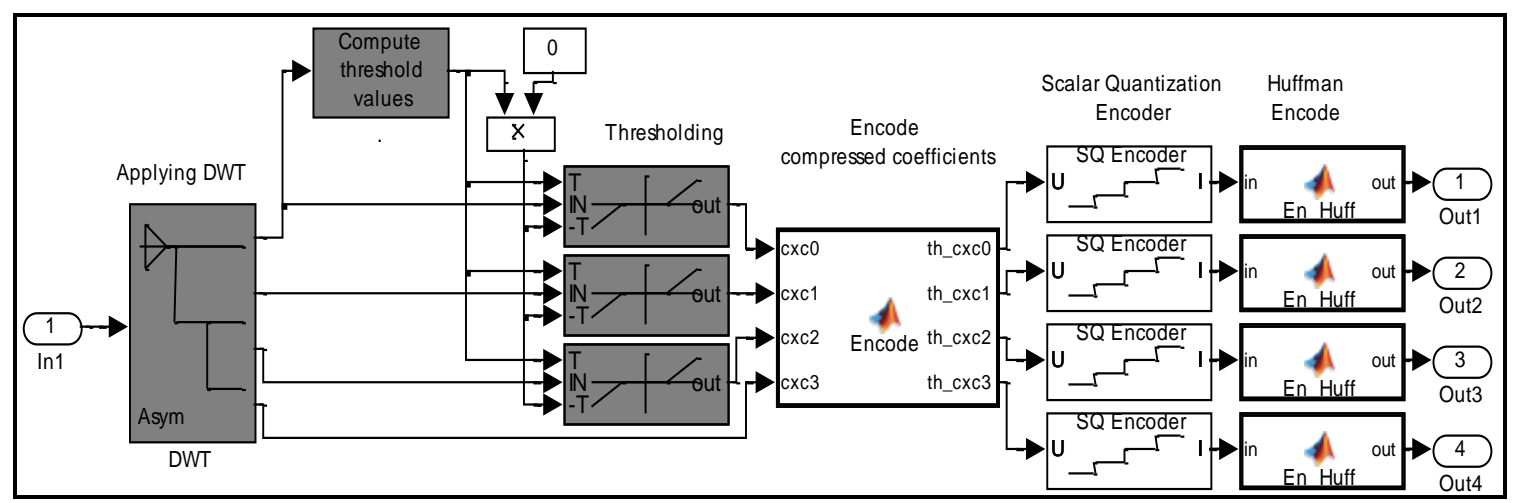

Fig. 11. Speech encoding process based on DWT using MATLAB/Simulink

Fig. 12 and Fig. 13 present the frame-base forward and inverse DWT architecture. The wavelet coefficients for each sub-band are computed using the recursive pyramid algorithm developed by S. Mallat, based on quadrature mirror filters (QMF).

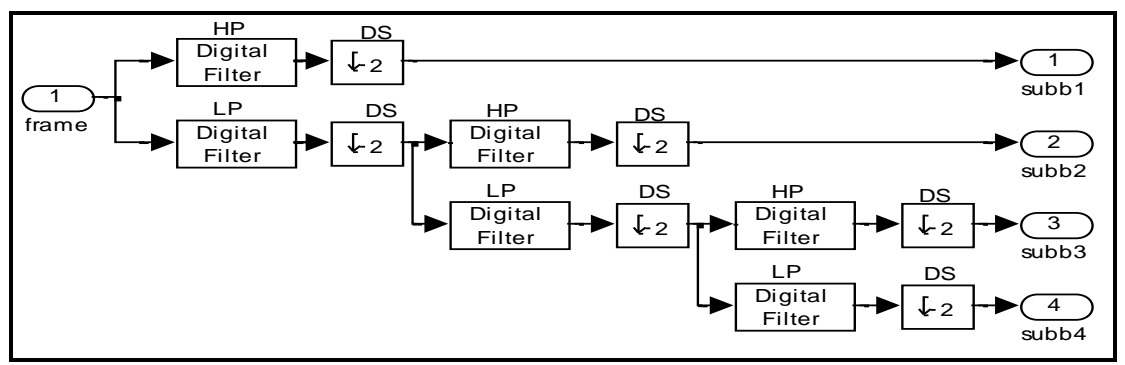

Fig. 12. Three-Level forward DWT using MATLAB/Simulink

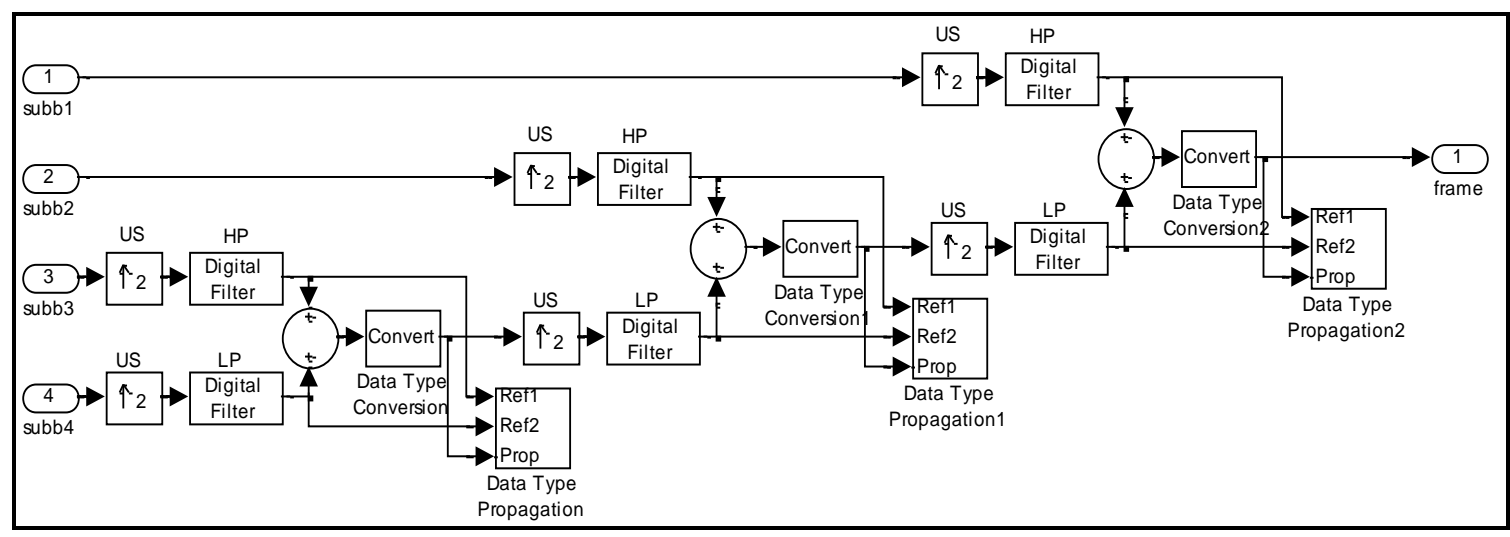

Fig. 13. Three-Level inverse DWT using MATLAB/Simulink 
Fig. 14 and Fig. 15 present the designed Simulink models to compute a global threshold values (11) and to apply a hard thresholding (9).

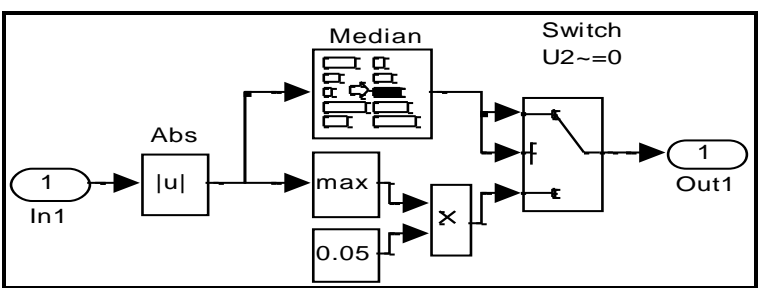

Fig. 14. Global threshold values computation using MATLAB/Simulink

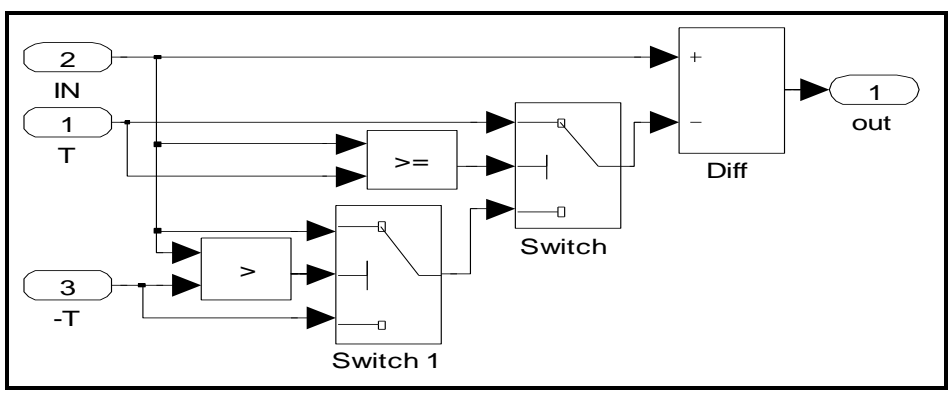

Fig. 15. Hard thresholding using MATLAB/Simulink

Finally, the DWT speech decoding algorithm can be summarized in four steps, as shown in Fig. 16:

1) Entropy decoding of the quantized sub-bands.
2) De-Quantization of the encoded sub-bands.

3) Decoding sub-bands.

4) Applying of the inverse DWT.

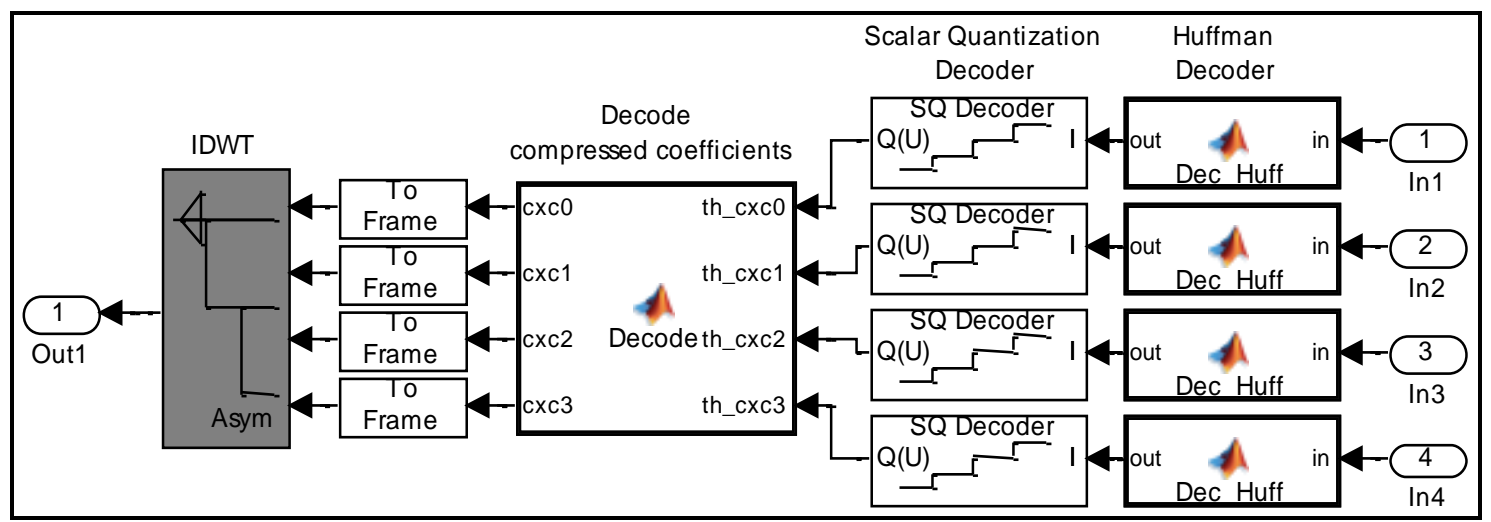

Fig. 16. Speech decoding process based on DWT using MATLAB/Simulink

In order to validate the performances of the optimized speech compression algorithm using DWT, the presents the number of cycles and the memory consumption are computed. Table 1 presents the number of cycles in MCPS and the memory consumption in kilobytes (KB) required to running the compression algorithm based on DWT, with and without VAD detector. In this work, the speech compression algorithm is applied to the speech frames of 256 samples. From the table 2 it can be noted that the total number of cycles decreases to about $49.6 \%$ by using the VAD module. So, the CPU speech and the memory consumption are fully satisfied the real time processing requirement on TMS320C6416 (CPU speed $=$ $1 \mathrm{GHz}, 512 \mathrm{~KB}$ of flash memory and $16 \mathrm{MB}$ of SRAM).

Table 2. Cycle count and memory consumption of speech compression algorithm using DWT

\begin{tabular}{|c|c|c|c|c|}
\hline Algorithms & Encoder (MCPS) & Decoder (MCPS) & Total (MCPS) & Memory consumption (Ko) \\
\hline DWT algorithm without VAD & 7.327 & 0.603 & 7.930 & 215.933 \\
\hline DWT algorithm with VAD & 3.563 & 0.378 & 3.941 & 276,344 \\
\hline
\end{tabular}

\section{CONCLUSION}

In this work, an optimized speech compression algorithm based on discrete wavelet transform is presented, and its real time implementation on fixed-point digital signal processor of Texas instruments is performed. It is shown that the adding of the voice activity module to the speech codec based on wavelets 
reduce the complexity of computation, the bit rate and achieved high speech coding performances.

\section{REFERENCES}

[1] O. Khalifa, S. Habib Harding, A. Hashim, "Compression using Wavelet Transform", Signal Processing: An International Journal, vol. 2, pp. 17-26, 2008.

[2] R.A. DeVore, B. Jawerth, B.J. Lucier, "Image compression through wavelet transform coding", IEEE Transactions on Information Theory, vol. 38, pp. 719-746, March 1992.

[3] H. Khalil, A. F. Atiya, S. Shaheen "Three-Dimensional Video Compression Using Subband/Wavelet Transform with Lower Buffering Requirements", IEEE transactions on image processing, vol. 8, June 1999.

[4] S. Ayat, M.T. Manzuri-Shalmani, R. Dianat, "An improved wavelet-based speech enhancement by using speech signal features", Computers \& Electrical Engineering, vol. 32, pp. 411-425, November 2006.

[5] B. Walczak, B. van den Bogaert, D. Luc Massart, "Application of Wavelet Packet Transform in Pattern Recognition of Near-IR Data", Analytical Chemistry, vol. 68, pp. 1742-1747, May 15, 1996.

[6] S. Ardhapurkar, R. Manthalkar, S. Gajre "Interpretation of Normal and Pathological ECG Beats using Multiresolution Wavelet Analysis", I.J. Information Technology and Computer Science, vol. 5, pp. 1-14, December 2012.

[7] G. Rajesh, A. Kumar and K. Ranjeet, "Speech Compression using Different Transform Techniques", IEEE International Conference on Computer and Communication Technology(ICCCT), pp. 146-151, 2011.

[8] Hatem Elaydi, "Speech compression using wavelets", 2010, http://site.iugaza.edu.ps/helaydi/files/2010/02/Elaydi.pdf

[9] S. Dusan , J. L. Flanagan, A. Karve, M. Balaraman, "Speech compression using polynomial approximation", IEEE Transactions on Audio, Speech, and Language Processing, vol. 15, pp. 387-395 , 2007.

[10] S. M. Joseph, "Spoken digit compression using wavelet packet”, IEEE international conference on signal and image processing (ICSIP-2010), pp. 255-259, 2010.

[11] Z. Dan, S. Ma, "Speech Compression with Best Wavelet Packet Transform and SPIHT Algorithm", International Conference on Computer Modeling and Simulation (ICCMS '10-2010), pp. 360-363, 2010.

[12] E. B. Fgee, W. J. Philips et W. Robertson, "Comparing audio compression using wavelet with other audio compression schemes", IEEE Proceedings, Electrical and Computer Engineering, vol. 2, pp. 698-701, 1999.

[13] M. Abdul Mawla, A. Najih, Abdul Rahman Ramli, Azizah Ibrahim, Syed A.R, "Comparing Speech Compression Using Wavelets With Other Speech Compression Schemes", Student Conference on Research and Development, Proceedings, pp. 55-58, 2003.

[14] N. Sriram, "Comparison of CELP speech coder with a wavelet method", Thesis, 2006.

[15] A. Grossman and J. Morlet, "Decomposition of Hardy functions into square integrable wavelets of constant shape”, SIAM J. Math. Anal., vol. 15, pp. 723-736, 1984.

[16] P. Gilles Lemariés-Rieusset, "Sur l'existence des analyses multi-résolutions en théorie des ondelettes", Revista Mathematica Ibero Americana, vol. 8, pp. 457-474, 1992.

[17] S. Mallat, "Multiresolution approximations and wavelet orthonormal bases of L ${ }^{2}(\mathrm{R})$ ", Trans. Amer. Math. Soc;. 315 , pp. 69-87, 1989.
[18] W. Kinsner, A. Langi, "Speech and Image Signal Compression with Wavelets", IEEE Wescanex Conference Proceedings, New York, NY, pp. 368-375, 1993.

[19] S. Kumar, V. K. Chaudhari, R. K. Singh,D. Varshney, “A New Algorithm for Voice Signal Compression (VSC) and Analysis Suitable for Limited Storage Devices Using MatLab", International Journal of Computer and Electrical Engineering, vol. 1, pp. 1793-8163, 2009.

[20] J. I. Agbinya, "Discrete wavelet transform techniques in speech processing", TENCON 96. Proceedings, IEEE TENCON. Digital Signal Processing Applications, vol. 2, pp. 514-519, 1996.

[21] D.L. Donoho, "De-Noising by softthresholding", IEEE Transaction on Inf. Theory, Vol. 41, pp.613-627, 1995.

[22] M. Misiti, Y. Misiti, G. Oppenheim et J. Poggi, "Matlab Wavelet Tool Box", The Math Works Inc., 2000.

[23] Y. Ephram and D. Malah, "Speech Enhancement Using aMinimum Mean-Square Error Short-Time Spectral Amplitude Estimator", IEEE Transaction on acoustics, speech and signal processing, vol. 32, pp. 11091121, 1984.

[24] G. Madre, E.H. Baghious, S. Azou et G. Burel, " Design of a variable rate algorithm for CS-ACELP Coder", IEEE International Conference on Communications (ICC'03), Anchorage, AK,USA, 2003.

[25] ITU-T Recommandation G.729 - Annex B, "Shéma de compression des silences pour la recommandation G.729 optimisé pour les terminaux conformes à la recommendation V.70", 1996.

[26] J. Garofolo, "Darpa Timit: Acoustic-phonetic Continuous Speech Corpus CD-ROM", US Department of Commerce, National Institute of Standards and Technology, 1993.

[27] MathWorks, "Real-Time Workshop For Use with SIMULINK", User's Guide, Version 6, June 2004.

[28] Texas instruments, "TMS320 DSP/BIOS", v5.42, User's Guide, spru423I, Août 2012.

\section{Authors' Profiles}

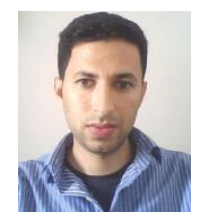

Noureddine Aloui: He received his Ph.D. degree in electronics from the Sciences Faculty of Tunis, and a researcher member in Innov'COM Laboratory, Sciences Faculty of Tunis, Tunisia.

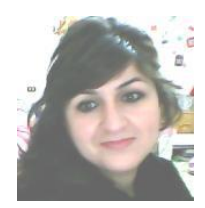

Souha Bousselmi: She is currently pursuing his Ph.D. in Signal Processing and a researcher Member in Innov'COM Laboratory Sciences Faculty of Tunis, Tunisia.

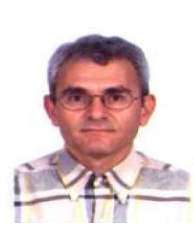

Adnane Cherif: is full Professor with the department of physics, Science Faculty of Tunis and responsible in Innov'COM Laboratory, Sciences Faculty of Tunis, Tunisia. 